\title{
Utility of magnetic resonance imaging in the evaluation of left ventricular thickening
}

\author{
Nicholas Fulton $^{1} \cdot$ Prabhakar Rajiah ${ }^{1,2}$
}

Received: 10 September 2016 /Revised: 12 February 2017 / Accepted: 14 February 2017 /Published online: 9 March 2017

(C) The Author(s) 2017. This article is published with open access at Springerlink.com

\begin{abstract}
Left ventricular (LV) thickening can be due to hypertrophy (concentric, asymmetric, eccentric) or remodelling (concentric or asymmetric). Pathological thickening may be caused by pressure overload, volume overload, infiltrative disorders, hypertrophic cardiomyopathy, athlete's heart or neoplastic infiltration. Magnetic resonance imaging (MRI) plays an important role in the comprehensive evaluation of LV thickening, including: establishing diagnosis, determining LV geometry, establishing aetiology, quantification, identifying prognostic factors, serial follow-up and treatment response. In this article, we review the aetiologies and pathophysiology of LV thickening, and demonstrate the comprehensive role of MRI in the evaluation of LV thickening.

\section{Teaching Points}

- MRI plays an important role in the evaluation of $L V$ thickening.

- LV thickening can be due to either hypertrophy or remodelling.

- Pathological thickening can be due to pressure/volume overload or infiltrative disorders.
\end{abstract}

Electronic supplementary material The online version of this article (doi:10.1007/s13244-017-0549-2) contains supplementary material, which is available to authorized users.

Prabhakar Rajiah

radprabhakar@gmail.com

1 Department of Radiology, University Hospital Case Medical Center, Cleveland, OH, USA

2 Department of Radiology Cardiothoracic Imaging, UT Southwestern Medical Center, E6.120 B, Mail code 9316, 5323 Harry Hines Boulevard, Dallas, TX 75390-8896, USA
Keywords Cardiac $\cdot$ MRI $\cdot$ LV thickening $\cdot$ Hypertension · Hypertrophy

\section{Introduction}

Left ventricular (LV) thickening is a commonly encountered finding in several imaging modalities. LV thickening can be either due to hypertrophy or remodelling. Pathological LV thickening may be caused by pressure/volume overload, infiltrative disorders, hypertrophic cardiomyopathy, athlete's heart or neoplastic infiltration (Table 1) [1]. LV hypertrophy (LVH) is characterized by increased length and width of the myocyte, as opposed to hyperplasia, which is characterized by generation of new myocytes. The development of hypertrophy is a complex process that involves myocardial stress, growth factors, cytokines, catecholamines and genetic abnormalities [2]. As per Laplace's law, the load in the myocardium is (pressure $\mathrm{x}$ radius)/ ( $2 \mathrm{x}$ wall thickness). In pressure overload, hypertrophy is caused by increased myosin heavy chain synthesis, whereas in volume overload, hypertrophy is caused by decreased myosin heavy chain degradation. Both these overloads also result in reprogramming of several genes, (e.g., ANP, ACE, SERCA-2, BBR, M2, etc.), which activate matrix metalloproteinase (MMPs) and increase the synthesis of collagen 1 resulting in fibrosis [3].

Regardless of the aetiology, LV thickening is an adverse prognostic determinant and requires treatment [1]. LV thickening is associated with higher risk of cardiovascular morbidity and mortality, independent of other traditional risk factors [4-6]. There is variability in these outcomes depending on the aetiology, LV geometry and ethnicity [1]. Regression of LVH has been shown to be associated with decreased risk and improved outcomes [7]. Imaging plays a vital role in the diagnosis and management of LV thickening. In this article, we review the comprehensive role 
Table 1 Causes of LV thickening

$\begin{array}{ll}\text { Pressure overload } & \text { Hypertension } \\ & \text { Aortic stenosis } \\ & \text { Coarctation } \\ & \text { Subaortic membrane } \\ \text { Volume overload } & \text { Aortic/mitral regurgitation } \\ & \text { Dilated cardiomyopathy } \\ \text { Infiltrative cardiomyopathies } & \text { Amyloidosis } \\ & \text { Anderson-Fabry disease } \\ & \text { Danon disease } \\ & \text { Eosinophilic heart disease } \\ & \text { Mucopolysaccharidoses } \\ & \text { Friedreich's ataxia } \\ & \text { Myocardial oxalosis } \\ & \text { Pompe disease } \\ & \text { Sarcoidosis } \\ \text { Iron overload } \\ \text { Non infiltrative cardiomyopathy } & \text { Hypertrophic cardiomyopathy } \\ \text { Adaptive response } & \text { Athlete's heart } \\ \text { Miscellaneous } & \text { Neoplastic infiltration }\end{array}$

of MRI in the evaluation of LV thickening and discuss the utility of MRI in several aetiologies of LV thickening.

\section{Imaging of left ventricular thickening}

Several imaging modalities are available and utilized in the evaluation of LV thickening, including echocardiography, computed tomography (CT) and MRI, each with their own advantages and disadvantages.

\section{Echocardiography}

Echocardiography is the most commonly used imaging modality in the evaluation of LV thickening. LV thickness and mass is reliably measured using the M-mode [8]. Systolic and diastolic functions are quantified using real time cine imaging. Although 2D echocardiography has been shown to be less accurate and reliable than MRI in the evaluation of the LV mass [9], 3D echocardiography has good accuracy and reproducibility [1]. Doppler technique is used to evaluate valvular and vascular lesions. Limitations of echocardiography include poor acoustic windows, attenuation and operator dependence [1].

\section{Computed tomography}

CT is also occasionally used to evaluate LV thickening, particularly in patients with contraindications for MRI. CT can outperform conventional echocardiography in diagnosing and quantifying the amount of LVH [10]. However, CT has been shown to overestimate the LV volume [11] and the reliability of ejection fraction is variable [12]. CT is also associated with ionizing radiation, potentially nephrotoxic contrast media and lower temporal resolution [13].

\section{MRI}

MRI is the most valuable imaging modality in the evaluation of LV thickening and enables comprehensive evaluation of LV thickening, including establishing diagnosis of LV thickening, determining LV geometry, establishing aetiology, quantification, identifying prognostic factors, serial followup and assessment of treatment response. In certain instances, particularly with suboptimal echocardiography, MRI is used in establishing the diagnosis of LV thickening. MRI can exquisitely demonstrate the specific LV geometric pattern, which has therapeutic and prognostic implications (see below). The tissue characterization capabilities of MRI help in establishing a specific aetiology of LV thickening, which is essential for determining the appropriate treatment strategy. MRI is considered the gold standard in quantification of LV mass as well as volumes and function due to its high accuracy and reproducibility [1]. This makes MRI ideal for serial measurements of LV mass in clinical scenarios as well as research trials to assess treatment response. MRI provides prognostic information based on mass, volumes, function, and fibrosis. MRI can evaluate other structures, including vasculature and valves [14]. Several MRI sequences are used in the evaluation of LV thickening. ECG-gated steady-state free precession (SSFP) cine sequence is used in morphological evaluation of the LV thickening as well as qualitative evaluation of ventricular function. LV mass, volumes and function can also be quantified from a stack of short axis slices by drawing endocardial and epicardial contours, both in the end-diastolic and end-systolic phases. Regional myocardial function can be accurately evaluated using several deformation techniques such as feature tracking, myocardial tagging, strain-encoded MRI (SENC) and displacement encoding with stimulated echoes (DENSE) [15]. Some of these techniques such as myocardial tagging require dedicated post processing software and additional time, which has precluded widespread clinical adoption of such techniques. Velocity-encoded phase-contrast (PC) sequence is used in the quantification of valvular lesions (stenosis, regurgitation), other obstructive lesions (coarctation) and shunts [16]. T2-weighted sequences (STIR, SSFP) are used in the evaluation of myocardial oedema. Post-contrast dynamic first pass perfusion imaging can identify perfusion defects (e.g., aortic stenosis, hypertrophic cardiomyopathy) [17]. Late gadolinium enhancement (LGE) sequences are used to detect scar/fibrosis, which helps in establishing aetiology of LV thickening as well as determining prognosis. T1 mapping sequences, both pre and post contrast, quantify the $\mathrm{T} 1$ values as well as extracellular volume (ECV), which is more sensitive in the detection of diffuse fibrosis than LGE. Additionally, T1 
mapping can identify deposits of amyloid, lipid and iron [18-20]. MR angiography (MRA) is useful in the evaluation of vascular abnormalities such as coarctation and renal arterial stenosis. The 4D flow sequences are useful in the assessment of flow characteristics such as direction and vortices, which may have a potential future clinical role.

\section{MRI parameters in $L V$ thickening}

On MRI, several measurements are utilized to quantify and characterize LV thickening. LV wall-thickness (LVWT) measured in an end-diastolic cine image is the most commonly used measurement. Normal LV myocardial thickness is $<11 \mathrm{~mm}$. Myocardial thickening is considered mild if it measures 11-13 mm, moderate if it measures $14-15 \mathrm{~mm}$ and severe if it measures $>15 \mathrm{~mm}$. $\mathbf{L V}$ mass (LVM) is quantified from end-diastolic phase images of short axis cine SSFP stack by drawing endocardial and epicardial contours. Using the modified Simpson's rule, the myocardial volume is obtained and multiplying this by myocardial density $(1.05 \mathrm{~g} / \mathrm{ml})$ gives the myocardial mass. An absolute mass $>184 \mathrm{~g}$ in males and $144 \mathrm{~g}$ in females, BMI-indexed mass $>91 \mathrm{~g} / \mathrm{m} 2$ in males and $>77 \mathrm{~g} / \mathrm{m} 2$ in females and height-indexed mass of $>83 \mathrm{~g} / \mathrm{m}$ in males and $>69 \mathrm{~g} / \mathrm{m}$ in females is considered LV thickening $[1,21,22]$. The height indexed mass is more reliable in obese patients, and also has been shown to correlate better with adverse cardiovascular events and mortality [23]. Other parameters that are crucial in classifying LV thickening are Relative wall mass (RWM), which is the ratio of LVM/ LVEDV (end-diastolic volume), normally $<1.16$ (Fig. 1); and Septal/lateral wall thickness, which is the ratio of thickness of septal and lateral segments. This ratio is normally 1 , but is abnormal in patients with asymmetric hypertrophy or remodelling.

\section{Geometrical patterns of $\mathrm{LV}$ thickening}

There are five different geometric patterns of LV thickening that can be exquisitely demonstrated using MRI, namely concentric hypertrophy, asymmetric hypertrophy, eccentric hypertrophy, concentric remodelling and asymmetric remodelling (Table 2). Broadly, the hypertrophic geometries are associated with increased LWM, while the remodelling geometries are associated with normal LWM (Fig. 1). Concentric hypertrophy is characterized by increased LVWT, LVWM and RWM, but with normal septal/lateral wall ratio. LV volume is normal or increased and EF is normal or decreased. This pattern is seen in pressure overload as well as infiltrative cardiomyopathies. Asymmetric hypertrophy is characterized by increased LVWT, LVWM, and RWM, but with an increased septal/lateral wall ratio. LV volume can be normal or decreased and EF can be normal or increased. Eccentric hypertrophy is characterized by increased LVWT and LVWM, but the RWM is subnormal $(<1.16)$ due to increased $\mathrm{LV}$ volume and the septal/lateral ratio is normal. $\mathrm{EF}$ is normal or decreased. Athlete's heart is classic example of eccentric hypertrophy presenting with a normal EF. Concentric remodelling is characterized by normal LVWT and LVWM, but the RWM is high due to low LV volume. Septal/lateral ratio and EF are normal. Asymmetric remodelling is characterized by normal LVWT and LVWM, but the RWM is high and EF is normal. Unlike concentric remodelling the septal/ lateral ratio is elevated. Concentric hypertrophy, eccentric hypertrophy and concentric remodelling are all associated with adverse events, with the highest risk seen in concentric hypertrophy [24].

\section{Etiologies of LV thickening}

The several aetiologies of LV thickening are discussed in Table 2. A flow chart to distinguish these entities is shown in Fig. 2. The comprehensive role of MRI in the evaluation of LV thickening is summarized in Table 3. The most common aetiologies are discussed in detail below.

\section{Pressure overload}

\section{HT}

Systemic hypertension (HT) can either be primary or secondary to lesions such as renal artery stenosis, adrenal tumours or coarctation. It has a prevalence of 20-30\% [1] with LVH seen in $30 \%$ of HT patients. LVH in HT is predominantly of a concentric hypertrophic pattern (Fig. 3a, b) [1] and HT is the most common cause of concentric LVH. Eccentric hypertrophy, concentric remodelling or a normal morphology can also be seen in HT [25]. The EF is preserved, and there is no systolic anterior motion of mitral valve (SAM) or LVOT obstruction. Left atrial enlargement and diastolic dysfunction may be present, both of which have prognostic value [1] LV dilation and systolic dysfunction may be seen in advanced cases with ventricular failure. Myocardial tagging shows depressed circumferential strain, most prominently in the septum [1]. No perfusion defect is identified per se, but may be seen due to associated coronary artery disease. LGE may be seen in $20-45 \%$ due to supply-demand mismatch, coronary microangiopathy or diffuse interstitial fibrosis $[1,26]$. LGE is seen in a mid-myocardial distribution either in the basal septal or inferolateral segments [1]. T1- mapping shows diffuse fibrosis, more common in eccentric LVH and is associated with systolic impairment, whereas concentric remodelling is associated only with abnormal aortic function [25]. Vascular 


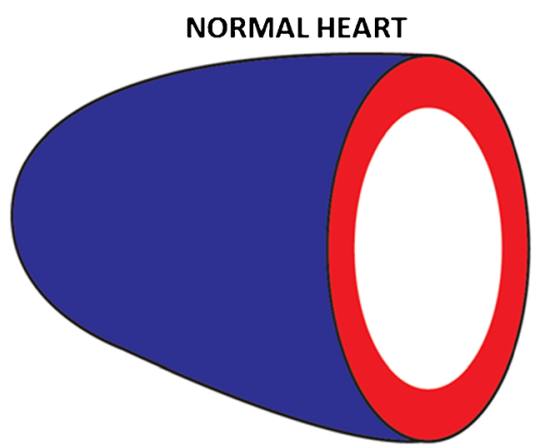

ECCENTRIC HYPERTROPHY
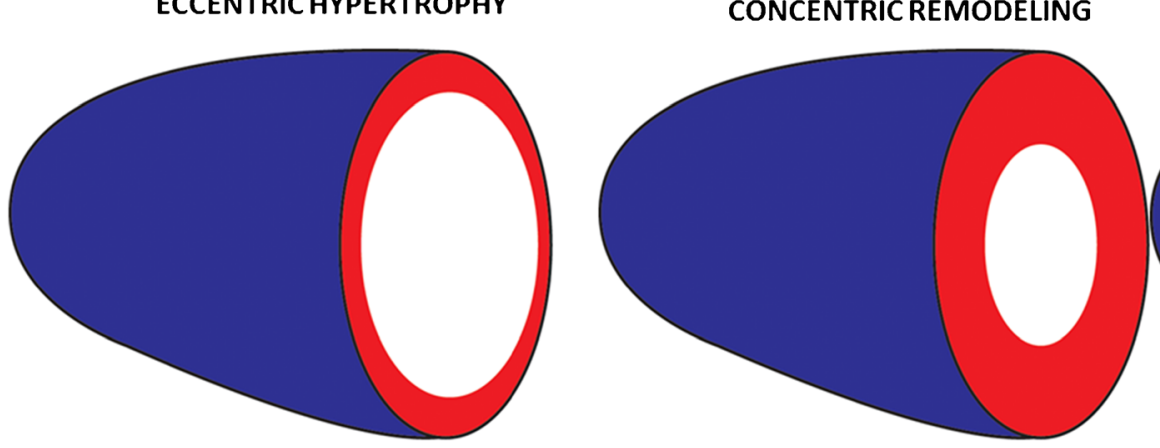

ASYMMETRIC HYPERTROPHY

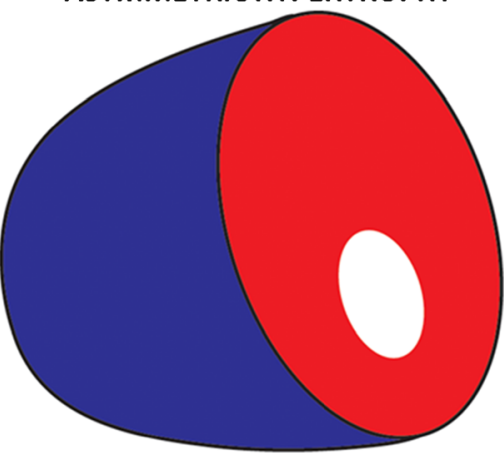

ASYMMETRIC REMODELING

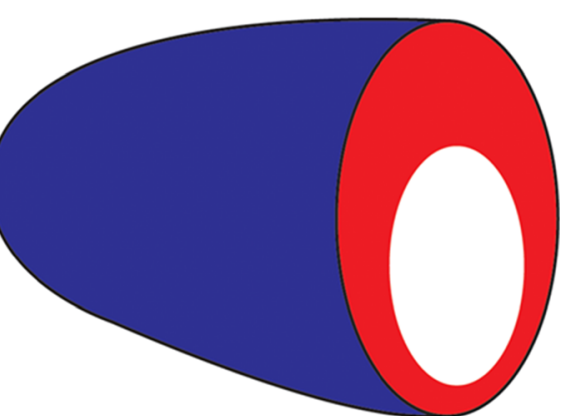

Fig. 1 Illustration showing the various types of left ventricular thickening. In a normal heart, the LV wall thickness, septal/lateral wall ratio, LV mass, LV volume, relative wall mass and ejection fraction are normal; in concentric hypertrophy, the wall thickness, LV mass and relative wall mass are increased. While septal/lateral wall ratio is normal, the $\mathrm{LV}$ volume is normal or increased and ejection fraction is normal or decreased; in asymmetric hypertrophy, the wall thickness, LV mass and relative wall mass are increased, but the septal/lateral wall ratio is increased. In eccentric hypertrophy, the wall thickness and septal/lateral wall ratio are normal, but LV mass and LV volume are increased, while ejection fraction and relative wall mass are decreased. In concentric remodelling, the relative wall mass is increased, the LV volume is decreased, but septal/lateral wall ratio, wall thickness, LV mass and ejection fraction are normal. In asymmetric remodelling, the relative wall mass is increased and LV volume is decreased. While septal/lateral wall ratio is increased, wall thickness, LV mass and EF are normal changes of HT seen in MRI include atheroma, ulcer, intramural hematoma, dissection and occlusive disease. These can be visualized in sequences such as high-resolution black-blood $\mathrm{T} 1$ and T2-weighted fast spin-echo as well as contrastenhanced 3d MRA sequences. MRI is valuable in establishing the aetiology of secondary hypertension, such as renal arterial stenosis/coarctation with MR angiography and adrenal adenoma/pheochromocytoma (Fig. 3c) with multi-sequence
MRI. MRI can be used to follow up response to therapy with decrease of LVH.

A diagnostic dilemma is to distinguish concentric hypertrophy due to HT from a concentric type of hypertrophic cardiomyopathy (HCM). On MRI, HT patients have normal or reduced EF, increased chamber volumes and occasional fibrosis, while HCM patients have normal or increased EF, normal chamber volumes and higher incidence of focal/diffuse

Table 2 Types of LV thickening and their distinguishing characteristics

\begin{tabular}{lllllll}
\hline & Wall thickness & $\begin{array}{l}\text { Septal/lateral } \\
\text { wall ratio }\end{array}$ & LV mass & LV volume & $\begin{array}{l}\text { Relative wall mass } \\
\text { (LVM/EDV) }\end{array}$ \\
\hline Normal & Normal & Normal & Normal & Normal & Normal & Normal \\
Concentric remodeling & Normal & Normal & Normal & Decreased & Increased & Normal \\
Asymmetric remodeling & Normal & Increased & Normal & Decreased & Increased & Normal \\
Concentric Hypertrophy & Increased & Normal & Increased & Normal/Increased & Increased & Normal/decreased \\
Eccentric hypertrophy & Normal & Normal & Increased & Increased & Normal/subnormal & Decreased \\
Asymmetric hypertrophy & Increased & Increased & Increased & Normal/decreased & Increased & Normal/increased \\
Infiltrative disease & Increased & Normal & Increased & Normal/decreased & Increased & Decreased/normal \\
\hline
\end{tabular}


Fig. 2 Flow chart showing the differentiation of various causes of LV thickening

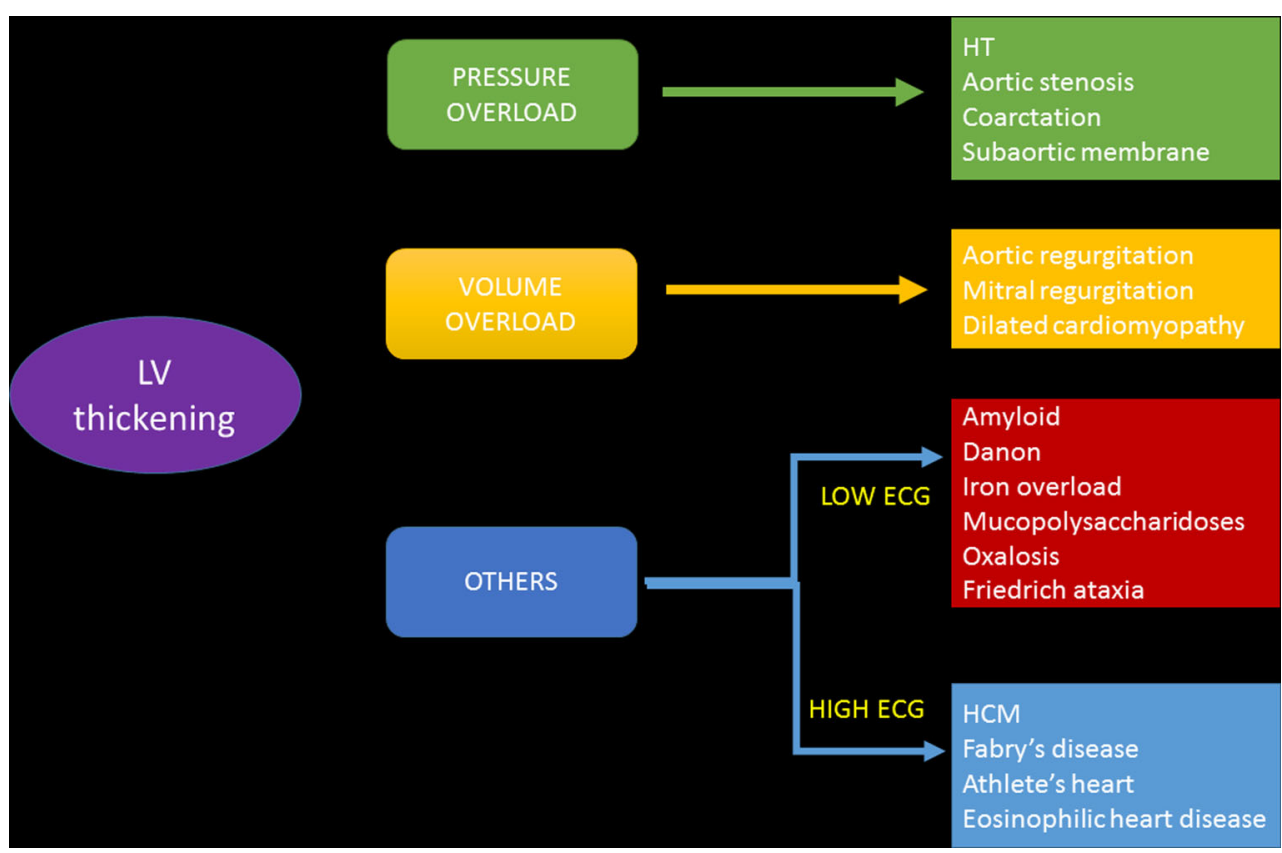

fibrosis. Although not widely available, strain imaging can help in distinguishing these entities, since HT patients have high wall stress and lower anteroseptal systolic strains, while HCM patients have decreased wall stress and longitudinal strain [2].

\section{Aortic stenosis}

Aortic stenosis (AS) is characterized by narrowing of the aortic valve, most commonly due to age related sclerosis, congenital bicuspid valve and rheumatic fever. It has a prevalence of 3-5\%. On MRI, the aortic leaflets are thickened and have low signal due to fibrosis/calcification. There is restricted opening of the valve leaflets in systole. The valve narrowing can be quantified by planimetry in dedicated short-axis views through the aortic valve (Fig. 4a). A dark dephasing jet is seen through the aortic valve in systole (Fig. 4b). The velocity of stenosis can be quantified using PC images obtained either perpendicular or parallel to the jet. The pressure gradient is derived by using the modified Bernoulli equation $\left(\Delta \mathrm{P}=4 \mathrm{v}^{2}\right)$ and the stenosis is graded as shown in Table 4 [27]. The aortic valve morphology in AS is variable, and can be trileaflet, bicuspid, unicuspid (Fig. 4c) or quadricuspid, best evaluated in cine SSFP or gradient-echo images. Compensatory concentric $\mathrm{LVH}$ is seen in $52 \%$ of $\mathrm{AS}$, which reduces the wall stress and maintains the cardiac output. Eventually, the left ventricle fails.

Although concentric LVH is the most common type, asymmetric hypertrophy, concentric remodelling, asymmetric remodelling, normal geometry and decompensation are also seen in AS [22]. There is no correlation between the severity of valve narrowing and extent of LVH [22]. Asymmetrical thickening is seen in $22 \%$ of AS, involving the basal and mid septum, which makes this indistinguishable from HCM [22]. Longitudinal strain may be diminished in early stages. In late stages, the $\mathrm{EF}$ is diminished along with LV dilation. Wall stress and diminished perfusion is seen in later stages due to ischemia. LGE may be seen due to fibrosis, likely due to myocardial ischemia caused by higher demand of LVH and high systolic pressure, and decreased myocardial perfusion due to lower aortic pressure, lower diastolic duration and absence of coronary vasodilation [28, 29]. LGE is most commonly seen in the basal segments, typically in a diffuse subendocardial distribution, but occasionally in a mid-myocardial distribution [30, 31]. LGE is associated with severe hypertrophy and adverse prognosis. MRI is also useful in the evaluation of patients being considered for transcutaneous aortic valve implantation (TAVI), especially in patients with contraindication for CT, by measuring the annulus, sinus of Valsalva, annulus to coronary ostial distance, fluoroscopic angulation plane and also the dimension and orientation of the peripheral access vessels [32].

\section{Aortic coarctation}

Aortic coarctation is discrete narrowing of the aorta, usually seen beyond the level of ductus arteriosus, but may also be seen proximal to it. More extensive areas of involvement result in hypoplasia. Coarctation has a prevalence of 0.01 to $0.03 \%$ and is seen in $0.2 \%$ of hypertensive adult patients [33]. Coarctation produces concentric LVH due to pressure overload. Hypertension is seen either due to mechanical obstruction, activation of the renin-angiotensin system, neural reasons and/or Goldblatt type phenomenon [1]. On MRI, the 
Table 3 Summary of the role of MRI in LV thickening

\begin{tabular}{|c|c|}
\hline Disease & Role of MRI \\
\hline General & $\begin{array}{l}\text { Establishing the presence of hypertrophy } \\
\text { Characterizing the geometry } \\
\text { Quantification of volumes, masses and function } \\
\text { Establishing aetiology } \\
\text { Fibrosis- Prognostic determinant } \\
\text { Evaluating regression }\end{array}$ \\
\hline Systemic hypertension & Secondary causes of hypertension- renovascular, adrenal lesion \\
\hline Aortic stenosis & $\begin{array}{l}\text { Planimetry- Aortic valve area } \\
\text { Phase contrast- Quantification of stenosis } \\
\text { Fibrosis } \\
\text { Evaluation for TAVI }\end{array}$ \\
\hline Coarctation & $\begin{array}{l}\text { Identification of coarctation/hypoplasia } \\
\text { Quantification of narrowing } \\
\text { Collaterals } \\
\text { Associated abnormalities }\end{array}$ \\
\hline Subaortic membrane & $\begin{array}{l}\text { Membrane- Location, size } \\
\text { Quantification of obstruction }\end{array}$ \\
\hline Aortic/mitral regurgitation & $\begin{array}{l}\text { Quantification of regurgitation } \\
\text { Volume quantification }\end{array}$ \\
\hline Dilated cardiomyopathy & $\begin{array}{l}\text { Quantification of volumes } \\
\text { Fibrosis }\end{array}$ \\
\hline Amyloidosis & $\begin{array}{l}\mathrm{T} 1 \text { kinetics and } \mathrm{T} 1 \text { mapping- Diagnosis and prognosis } \\
\text { Late gadolinium enhancement }\end{array}$ \\
\hline Danon disease & Fibrosis \\
\hline Hypertrophic cardiomyopathy & $\begin{array}{l}\text { Pattern of HCM } \\
\text { Quantification of obstruction } \\
\text { Mitral valve abnormalities- SAM, elongated anterior leaflet } \\
\text { Papillary muscle abnormalities } \\
\text { Fibrosis }\end{array}$ \\
\hline Athlete's heart & $\begin{array}{l}\text { Quantification of volumes } \\
\text { Response to deconditioning }\end{array}$ \\
\hline $\begin{array}{l}\text { Anderson Fabry disease } \\
\text { Iron overload cardiomyopathy }\end{array}$ & $\begin{array}{l}\text { T1 mapping allows detection of fibrosis and lipid deposition } \\
\text { Myocardial iron quantification }\end{array}$ \\
\hline
\end{tabular}
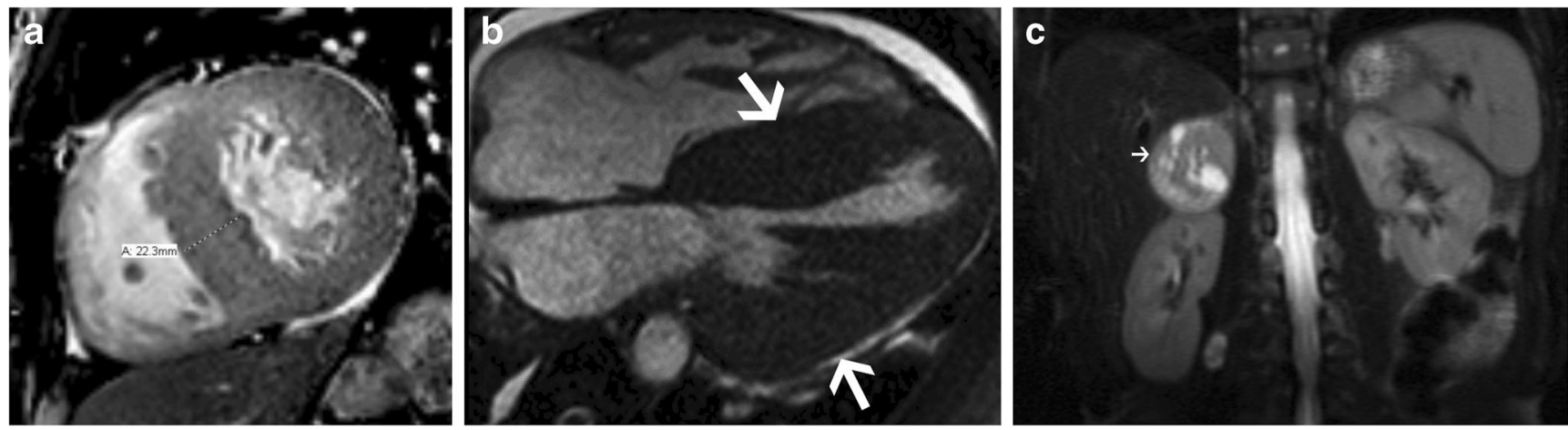

Fig. 3 a Short axis cine-SSFP image in a patient with hypertension shows severe concentric LV hypertrophy with maximal thickness of $2.2 \mathrm{~cm}$ in the mid septum in end diastole. b 4-chamber cine SSFP image in another patient with HT shows severe concentric LV hypertrophy. c
Coronal T1-weighted image through the upper abdomen after gadolinium administration shows an intensely enhancing mass in the upper abdomen (arrow), consistent with a pheochromocytoma 

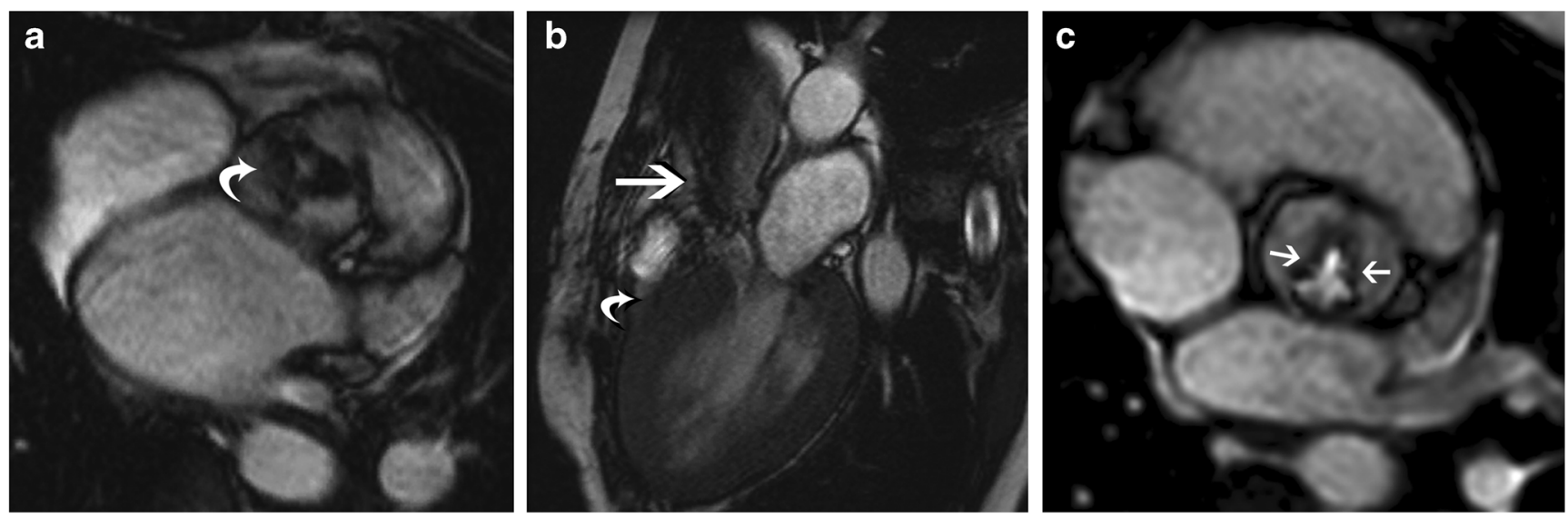

Fig. 4 a Short axis cine SSFP image through the aortic valve shows thickening of the valve leaflets (arrow) and restricted systolic opening. The valve area was measured to be $0.5 \mathrm{~cm} 2$, consistent with severe aortic stenosis. b 3-chamber cine SSFP sequence in the same patient shows restricted systolic opening of the aortic valve and flow acceleration

through the aortic valve (straight arrow), consistent with aortic stenosis Concentric LV hypertrophy is also seen (curved arrow). c Short axis cine SSFP image through the aortic valve shows a unicuspid aortic valve with thickened leaflets (arrows) and restricted opening consistent with aortic stenosis

coarctation and collateral vessels are evaluated and quantified using cine-SSFP or MR angiographic images (Fig. 5a). MRI can assess the presence and direction of flow in collaterals. The flow velocity, flow volume and pressure gradients at the coarctation site can be measured using a velocity-encoded PC sequence (Fig. 5b). Presence of higher flow in the distal segment of descending thoracic aorta compared to the proximal segment is an indicator of hemodynamically significant obstruction due to filling of the distal aorta by collateral vessels. LVH is seen in $20-40 \%$ of coarctation [33, 34]. Subendocardial ischemia and abnormal arterial stiffness are also seen [1]. Associations of coarctation include bicuspid aortic valve, ventricular septal defect (VSD), hypoplastic left heart and subaortic stenosis, all of which can be evaluated using MRI. MRI is used for preoperative/interventional planning and post-treatment assessment of complications.

\section{Subaortic membrane}

The subaortic membrane is a discrete fibrous membrane, muscular narrowing or a combination of both. It usually involves the LV outflow tract (LVOT), but may also involve the septum, anterior mitral leaflet or the aortic valve. Common associations of subaortic membrane include bicuspid aortic valve, AV canal, Shone's complex, tunnel subaortic stenosis and VSD. On MRI, focal discrete narrowing of the LVOT by a membrane is identified (Fig. 6) in cine SSFP and 3D whole heart SSFP images. Flow acceleration and dephasing is also identified (Fig. 6) in cine images, which can be quantified by using PC images. A peak gradient $>50 \mathrm{~mm} \mathrm{Hg}$ is considered to be severe obstruction. Concentric LVH is present due to pressure overload and subendocardial ischemia may be seen. Complications include infective endocarditis and aortic regurgitation due to structural valvular damage. Treatment is with surgical resection when there is either hemodynamically significant obstruction or aortic valvular involvement [35].

\section{Volume overload}

Volume overload conditions such as long-standing aortic or mitral regurgitation and dilated cardiomyopathy lead to complex forms of adaptation resulting in eccentric LV hypertrophy. The LVWT and LVWM are increased, but the RWM is lower than normal due to ventricular dilation. MRI can quantify the regurgitation as well as its secondary effects on the ventricle including abnormal chamber size and function. In dilated cardiomyopathy, the LV is dilated and there is global systolic dysfunction. A nonischemic pattern of LGE may be seen, in a mid-myocardial or subepicardial distribution

Table 4 Grades of aortic stenosis [27]

\begin{tabular}{llllll}
\hline & Peak velocity $(\mathrm{m} / \mathrm{s})$ & Mean gradient & Valve area & Indexed valve area & Velocity ratio \\
\hline Mild & $2.6-2.9$ & $<20^{\mathrm{a}}\left(<30^{\mathrm{b}}\right) \mathrm{mm} \mathrm{Hg}$ & $<1.5 \mathrm{~cm} 2$ & $0.85 \mathrm{~cm} 2 / \mathrm{m} 2$ & $>0.5$ \\
Moderate & $3.0-4.0$ & $20-40^{\mathrm{a}}\left(30-50^{\mathrm{b}}\right) \mathrm{mm} \mathrm{Hg}$ & $1-1.5 \mathrm{~cm} 2$ & $0.60-0.85 \mathrm{~cm} 2 / \mathrm{m} 2$ & $0.25-0.5$ \\
Severe & $>4.0$ & $>40^{\mathrm{a}}\left(50^{\mathrm{b}}\right) \mathrm{mm} \mathrm{Hg}$ & $<1 \mathrm{~cm} 2$ & $<0.6 \mathrm{~cm} 2 / \mathrm{m} 2$ & $<0.25$ \\
\hline
\end{tabular}

a AHA/ACC guidelines

${ }^{\mathrm{b}}$ ESC guidelines 
Fig. 5 a Sagittal volume rendered 3D image in a patient with concentric LV thickening shows severe coarctation of the aortic arch (straight arrow) and multiple collateral vessels(curved arrows). b Velocity encoded phase contrast image in the sagittal plane in another patient shows flow acceleration through the aortic arch at the site of coarctation, which was quantified and the pressure gradient was estimated to be $20 \mathrm{~mm} \mathrm{Hg}$
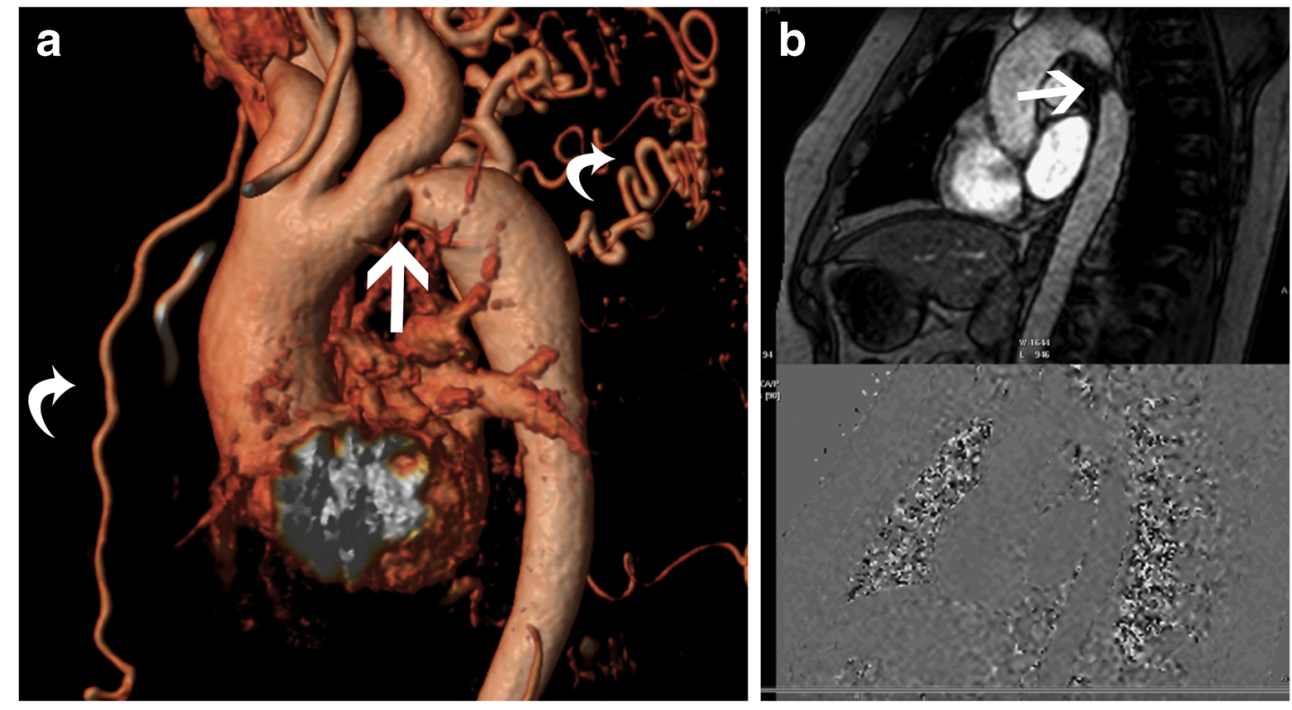

depending on the underlying aetiology [36]. A linear midmyocardial pattern has been described in idiopathic dilated cardiomyopathy [36].

\section{Infiltrative cardiomyopathies}

Infiltrative cardiomyopathies are characterized by the deposition of abnormal substances in the myocardium resulting in diminished ventricular filling. Some of these entities cause LV thickening, whereas other entities predominantly manifest as chamber dilation and wall thinning but occasionally may manifest with wall thickening/remodelling. The former category includes cardiac amyloidosis, Fabry's disease, Danon disease, eosinophilic heart disease, Friedreich ataxia, oxalosis and mucopolysaccaridoses, while the latter category includes sarcoidosis and iron overload cardiomyopathy [37].

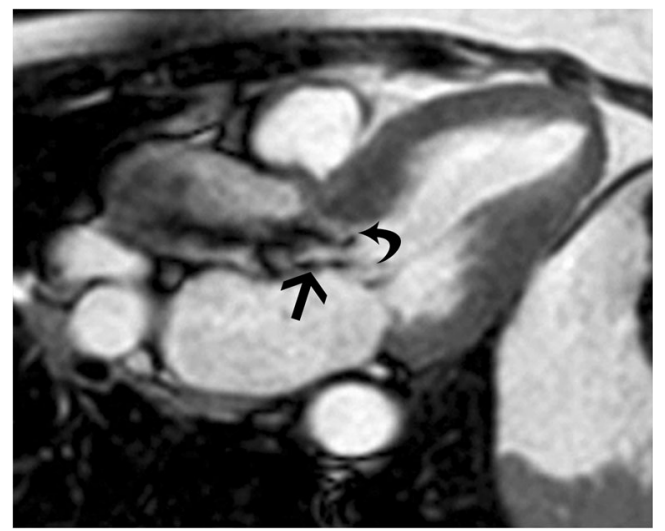

Fig. 6 3-chamber cine SSFP image shows concentric LV thickening. There is flow acceleration in the LVOT below the level of the aortic valve (straight arrow). There is a linear discrete membrane at the LVOT (curved arrow), which is responsible for the flow acceleration, consistent with a subaortic membrane

\section{Cardiac amyloidosis}

Cardiac amyloidosis is characterized by the deposition of $\beta$ pleated amyloid proteins, with an estimated prevalence of $0.001 \%$. In the AL type, cardiac involvement is seen in 33\%; in the AA type, cardiac involvement is unusual and in TTRtype; there are specific gene mutations [38]. On MRI, concentric LV thickening is seen, along with thickening of the RV, atria, interatrial septum and valves (Fig. 7a). Biatrial dilation and decreased LV volumes are also present. Longitudinal strain is decreased in the early stages and eventually EF is also decreased. There is no SAM or LVOT obstruction. Perfusion is usually normal, but a subendocardial defect may be seen. Due to interstitial expansion from amyloid deposition, LGE is seen in $69-97 \%$ of all cardiac amyloidosis patients, $100 \%$ of TTR type and $64 \%$ of AL type [38]. LGE is usually seen in a global transmural or subendocardial pattern (83\%) (Fig. 7b), but occasionally may be focal and patchy $(6 \%)$. In $8 \%$ of patients, a suboptimal myocardial nulling (8\%) may be the only finding. LGE is seen in MRI in 47\% of confirmed cardiac amyloidosis patients without echocardiographic findings [39]. LGE is associated with NYHA functional class, ECG voltage, LVM index, RV wall thickness, troponin T and BNP levels [37]. Alteration in $\mathrm{T} 1$ kinetics can be demonstrated in the T1-scout images, with nulling of the myocardium occurring at the same time or even before nulling of the blood pool (Fig. 7c) (which is the reverse of the normal pattern where the normal myocardium always nulls after the blood pool). At 2 minutes post contrast, a T1 difference between subepicardium and subendocardium of $<23$ milliseconds ( $\mathrm{ms}$ ) is a predictor of mortality with $85 \%$ accuracy [38]. Native T1 mapping shows elevated T1 values, which is an early indicator and direct marker of cardiac amyloid [40]. Higher ECV has been shown with post contrast T1 mapping, which directly correlates with LGE, indexed LV mass and other clinical adverse prognostic factors [41]. 


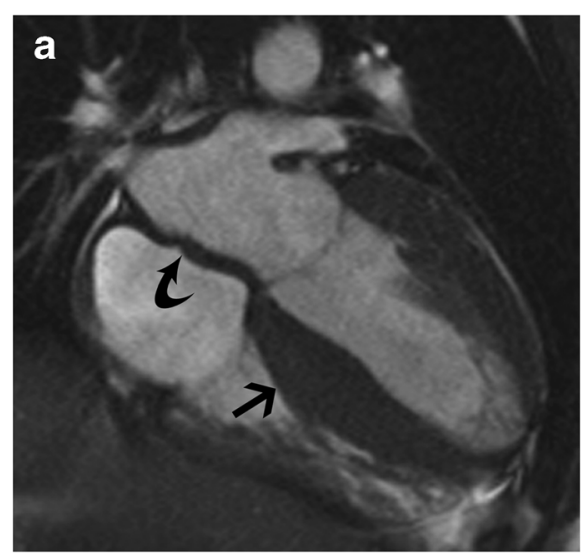

Fig. 7 a 4-chamber SSFP image in a patient with cardiac amyloidosis shows concentric LV thickening (straight arrow). There is also thickening of the interatrial septum (curved arrow). b Post contrast T1-w inversion recovery gradient echo image shows diffuse sub-endocardial to mid myocardial LGE of the ventricles (straight arrow), interatrial septum (curved arrow) and the atrial walls (arrowhead). c Short-axis TI scout

\section{Anderson-Fabry's disease}

Anderson-Fabry's disease is an X-linked storage disorder characterized by the accumulation of glycosphingolipid due to a deficiency of $\alpha$-galactosidase enzyme. In the early stages, there is concentric LV thickening, prominent papillary muscles, preserved EF and diastolic dysfunction, which then progresses to intramural replacement fibrosis, regional wall motion abnormalities [42] and arrhythmias. On MRI, LV thickening is present, usually concentric but may be asymmetric. Fabry's disease accounts for 3\% of all LVH [43]. Fabry's disease has been shown to be the aetiology in 6-12\% of patients presenting with suspected symmetric HCM [44]. LGE is seen in up to $50 \%$ of patients with Fabry's disease due to cardiac infiltration [45]. LGE in Fabry's disease is typically seen in the basal inferolateral wall in a mid-myocardial or subepicardial pattern (Fig. 8). However, in HCM, LGE is seen in hypertrophied as well as non-hypertrophied segments, usually in a mid-myocardial distribution or at RV insertion points [45]. LGE is associated with poor prognosis. Native T1 values are low in Fabry's disease due to presence of lipids, whereas in $\mathrm{HCM}$ it is high due to fibrosis. Elevated T2, RV involvement, valve thickening/dysfunction and decreased longitudinal systolic motion have also been reported in Fabry's disease.

\section{Danon disease}

Danon disease is an $\mathrm{X}$-linked dominant lysosomal storage disorder characterized by mutation of lysosomal associated protein2 (LAMP2). Cardiac manifestation of Danon disease includes concentric LV thickening, cardiac failure and Wolff-ParkinsonWhite syndrome. Other features are myopathy, elevated creatine kinase, eye abnormalities and mental retardation. On MRI, concentric LV thickening is present. Danon disease has been

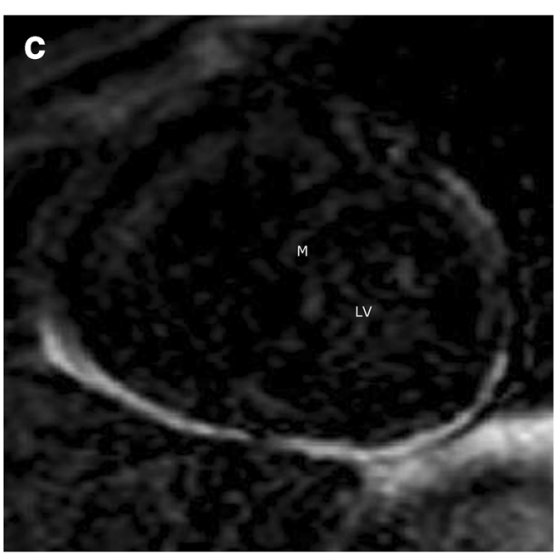

(Look Locker sequence image) at the level of mid-ventricular septum in a patient with cardiac amyloidosis. At an inversion time of 150 milliseconds, both the blood pool (LV) and myocardium (M) are dark. This is due to diffuse myocardial infiltration with amyloid, which takes up gadolinium and results in $\mathrm{T} 1$ shortening

confirmed as the correct diagnosis in $4 \%$ of patients initially diagnosed as HCM [46]. Myocardial oedema and perfusion defect are occasionally seen. LGE is seen in a non-ischemic pattern in a mid-myocardial distribution [47] (Fig. 9), but occasionally subendocardial and transmural distribution may be seen [48]. There is often sparing of the septum between the RV insertion points [49]. Involvement of the heart is associated with poor prognosis. Due to the risk of sudden cardiac death, implantable cardioverter defibrillator (ICD) placement or cardiac transplantation are performed.

\section{Eosinophilic endocarditis}

Eosinophilic endocarditis (endomyocardial fibrosis, Löeffler endomyocarditis) is a part of the spectrum of hyperesosinophilic

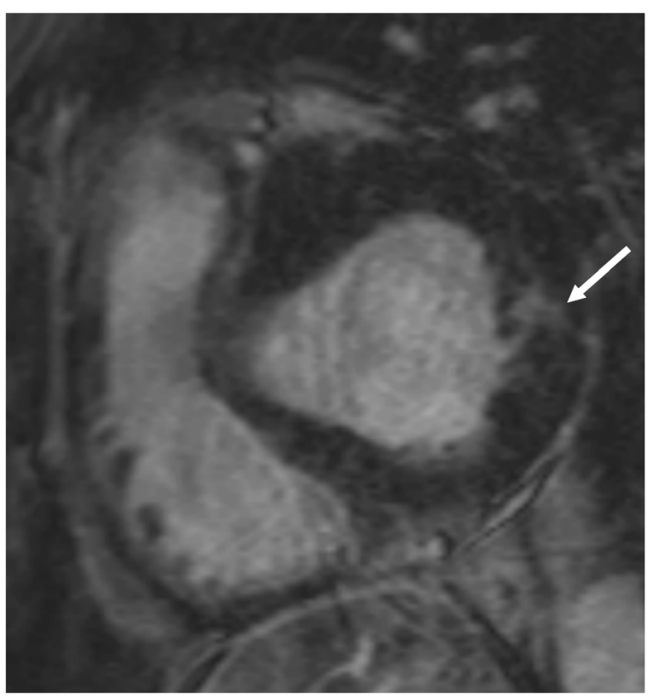

Fig. 8 Short-axis post contrast T1-w inversion recovery gradient echo MRI sequence shows patchy mid myocardial LGE in the basal lateral (straight arrow) in a patient with Anderson Fabry's disease 


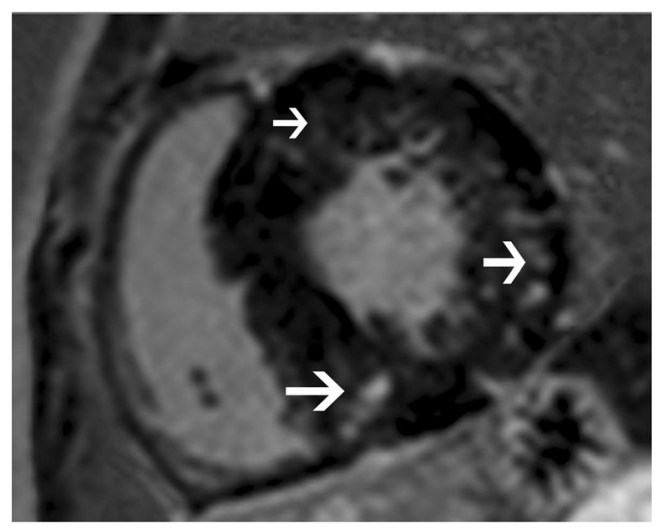

Fig. 9 Short-axis post-contrast T1-w inversion recovery gradient echo image shows patchy mid myocardial LGE (arrows) of severely thickened myocardium in a patient with Danon disease

syndrome, which is characterized by elevated eosinophils $>$ $1500 / \mu \mathrm{L}>6$ months and organ dysfunction. The heart is the most commonly involved organ and cardiac involvement is associated with adverse prognosis. There are three stages of cardiac involvement. In the acute necrotic stage, there is eosinophilic infiltration of the endocardium and myocardium. This is followed by the thrombotic-necrotic stage, which is associated with ventricular damage. In the late fibrotic stage, there is diffuse fibrous tissue lining the endocardium often extending from the LVOT to the apex. On MRI, there is thickening of the LV apex, which can be confused with apical HCM. A high T2 signal may be seen in the apical endocardium. Diffuse subendocardial perfusion defect may be present. On LGE, a triple layered pattern of enhancement is a characteristic finding of eosinophilic endocarditis. This includes an inner dark layer due to non-enhancing thrombus, a middle bright layer due to LGE from fibrous tissue and an outer dark layer of normally nulled myocardium (Fig. 10). Patchy intramyocardial LGE may also be present $[50,51]$. Associated wall motion abnormalities and dilated left atrium may also be seen. Treatment is surgical resection of the fibrous tissue.

\section{Iron overload cardiomyopathy}

Iron overload cardiomyopathy is characterized by excess myocardial iron deposition, secondary to hemochromatosis, sickle cell disease, thalassemia, multiple transfusions or increased gastrointestinal (GI) absorption of iron [52]. It has an estimated prevalence of $0.0001 \%$. There are two phenotypes of iron overload cardiomyopathy, the restrictive type with preserved $\mathrm{EF}$, diastolic dysfunction, LA/RV dilation and pulmonary hypertension; and the dilated type with LV remodelling, chamber dilation and decreased EF [53]. Eccentric hypertrophy, high EF and high cardiac output may also be seen [53]. Iron overload cardiomyopathy can result in ventricular failure, arrhythmia and mortality [52]. Hence, it is the most important determinant of survival in patients with

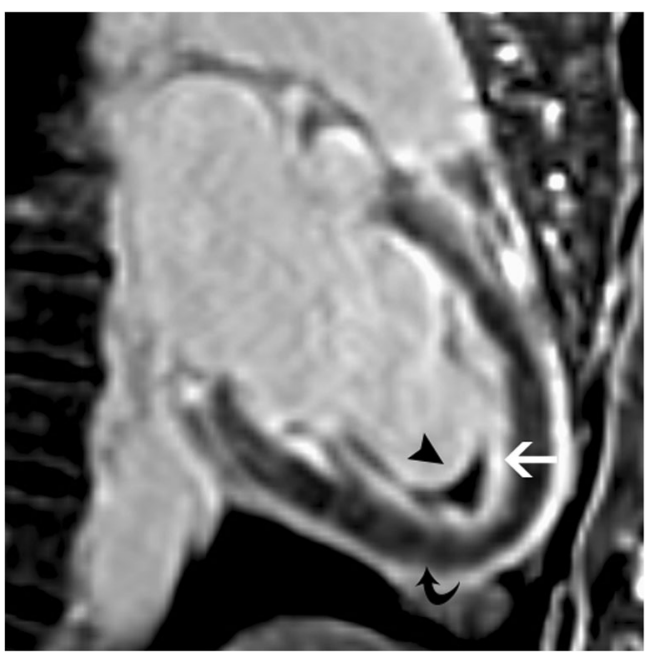

Fig. 10 3-chamber post contrast $\mathrm{T} 1-\mathrm{w}$ inversion recovery image shows a triple layered pattern of enhancement with an inner layer of dark thrombus (arrowhead), middle layer of LGE due to enhancing fibrous tissue (straight arrow) and an outer layer of dark normal myocardium (curved arrow)

thalassemia or multiple transfusions. It is imperative to make the diagnosis of iron overload cardiomyopathy prior to the development of overt cardiac failure, since early initiation of chelation therapy can improve the outcome and reduce adverse events [52]. On MRI, multi-echo gradient echo images are obtained in the same slice at different TE values, and the mean myocardial signal intensity at each TE level is measured (Fig. 11). A curve fit is used to obtain myocardial T2. Normal myocardial T2* is $33 \pm 6.5$ milliseconds. Myocardial iron deposition is diagnosed when $\mathrm{T} 2 *$ is $<20$ milliseconds and is considered severe, when $\mathrm{T} 2 *$ is $<10$ milliseconds [54].

\section{Sarcoidosis}

Sarcoidosis is a systemic disorder characterized by the presence of non-caseating granulomas. Cardiac involvement is seen in 5-20\% of patients with sarcoidosis. In the acute stage, diffuse myocardial thickening may be seen due to diffuse infiltration. Regional wall motion abnormalities, myocardial oedema and LGE are also seen. LGE is seen in a mid-myocardial or subepicardial distribution. On STIR, confluent granulomas may be seen with a central low signal of fibrous tissue and a peripheral rim of high signal with a nodular component, more common in the basal septum than the lateral wall. In the chronic stage, patchy areas of mid-myocardial to subepicardial LGE may be seen with associated normal or thinned myocardium (Fig. 12) [55]. No oedema is seen in the chronic stage.

\section{Uncommon conditions}

Myocardial oxalosis is characterized by the deposition of oxalic acid crystals in the heart due to its higher production. 


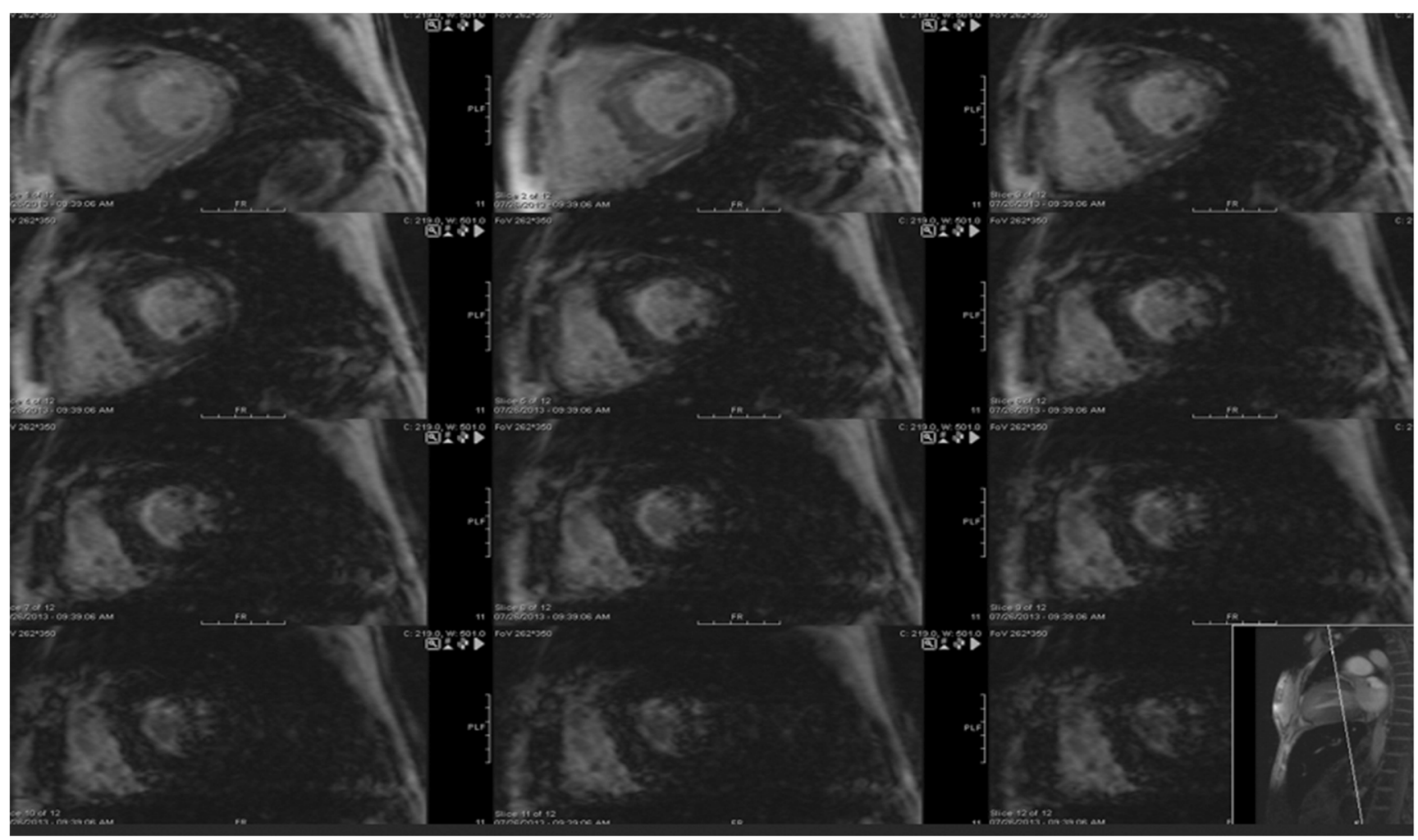

Fig. 11 Short-axis multi-echo gradient echo T2 weighted images obtained at different TEs, shows that the myocardium gets progressively darker with increasing TEs, with complete darkness at TE of 14.2 milliseconds, indicating significant iron deposition

Clinical presentation includes LVH, heart block and conduction abnormalities. On MRI, there is concentric left and right ventricular thickening. Diastolic dysfunction may be seen. Friedreich ataxia is characterized by mitochondrial ion accumulation, with cardiomyopathy seen in $63 \%$. On MRI,

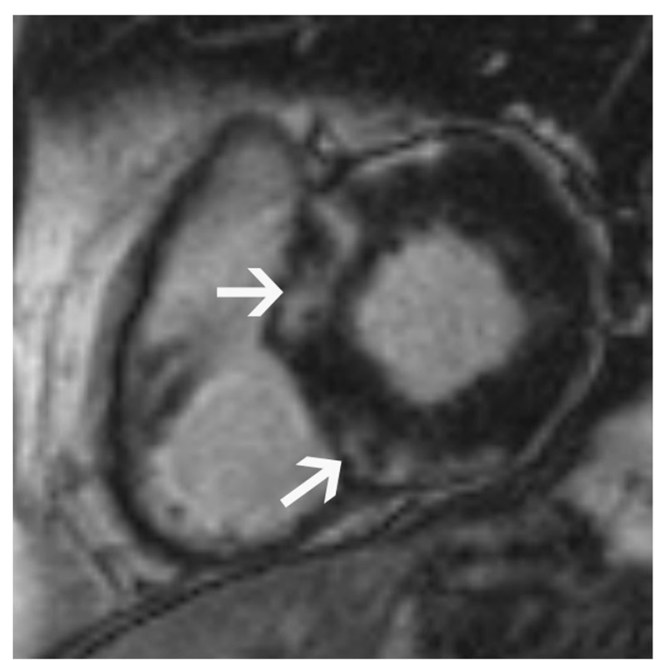

Fig. 12 Short-axis post-contrast T1-w inversion recovery gradient echo image shows mild concentric LV thickening and patchy areas of mid myocardial and subepicardial LGE (arrows) in a patient with cardiac sarcoidosis concentric or asymmetric LVH is seen, without evidence of LVOT obstruction. Diastolic dysfunction and fibrosis may be seen [37]. Mucopolysaccharidoses can present with variable MRI appearance depending on the phenotype, including asymmetrical septal thickening, mitral and or aortic valve stenosis/insufficiency with normal EF [37]. In obesity, LVH with high LVM, LVEDV and RWM is seen, with preserved EF [56] which correlates with the body mass. LVH with higher LVM with normal EF is also seen in DM Type II, correlating with BMI and HbAlc [57].

\section{Hypertrophic cardiomyopathy}

Hypertrophic cardiomyopathy (HCM) is characterized by myocardial hypertrophy without an underlying cause such as HT or AS. It is caused by sarcomeric gene mutations and has variable phenotypic expression. It has a prevalence of $0.2 \%$, and LVH is seen in $60 \%$ of these patients [1]. The most common pattern of HCM is the asymmetrical hypertrophy, which typically involves the basal ventricular septum (Fig. 13a), but occasionally may involve the apical region (Yamaguchi type) (Fig. 13b), mid-ventricular region or even the lateral wall. Spiral and mass-like types (Fig. 13c) have also been described. The concentric type of HCM is rarely seen. On 

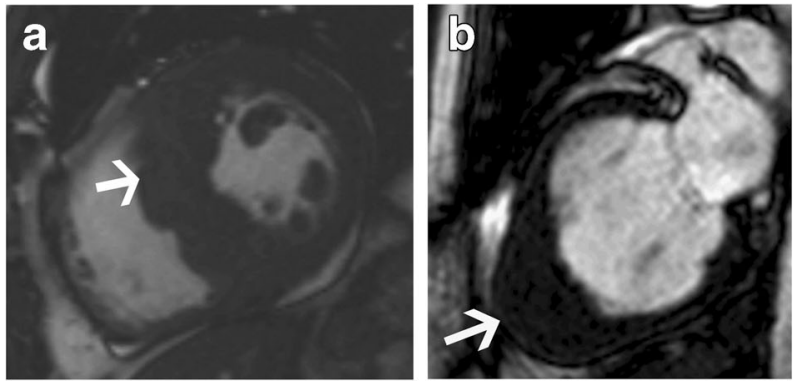

Fig. 13 a Short-axis SSFP image shows severe asymmetric hypertrophy of the mid-ventricular septum, while the lateral, anterior and inferior segments appear to be of normal thickness. b 2-chamber SSFP image shows severe thickening of the apical LV segments, consistent with apical variant (Yamaguchi type) of hypertrophic cardiomyopathy. c 3chamber cine SSFP image shows extensive mass-like appearance of a
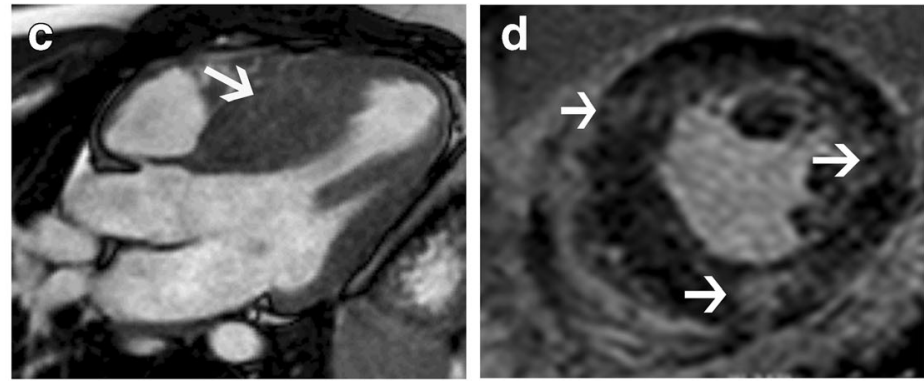

focal hypertrophic cardiomyopathy involving the mid-ventricular septum (arrow). d Short axis post contrast inversion recovery SSFP sequences shows patchy, fine mid-myocardial LGE of the hypertrophied septum, especially at RV insertion points (arrows) that are consistent with interstitial fibrosis in a pattern that is specific for hypertrophic cardiomyopathy
MRI, there is asymmetric hypertrophy, with a septal/lateral wall $>1$. EF is either normal or hyperdynamic. Ventricular chamber size is normal but may be dilated in the end stages. In the obstructive type (HOCM), there is flow acceleration in the LVOT due to narrowing, which results in systolic dephasing. This can be quantified using velocity encoded PC imaging. SAM augments this obstruction and also results in eccentric posteriorly mitral regurgitation and left atrial enlargement. MRI can also help in evaluating papillary muscle abnormalities such as abnormal insertion, position and mobility which can augment the obstruction or may be the only cause of obstruction. Occasionally these abnormalities are seen without significant hypertrophy. While the classical HCM may need surgical myectomy or septal alcohol ablation, papillary muscle abnormalities require specific surgeries such as reimplantation of papillary muscles [58]. Mitral valve abnormalities, such as elongation, can also be seen in MRI [59], and these require mitral valve surgery. Stress perfusion defects may be seen. LGE is seen in $40-80 \%$ of HCM patients, either due to myocyte disarray, dysplasia of intramyocardial arterioles, interstitial fibrosis, or other types of fibrosis (plexiform, pericellular, perivascular, or substitution) [60]. LGE is typically patchy and seen in a mid-myocardial distribution, both in hypertrophied and non-hypertrophied areas, and also at RV insertion points (Fig. 13d). Detection and quantification of LGE is the most significant contribution of MRI in HCM, since the presence of LGE has been shown to be associated with adverse cardiovascular events including arrhythmias and sudden cardiac death, often warranting ICD placement [60-62]. LGE often underestimates the amount of fibrosis in $\mathrm{HCM}$, since the fibrosis is diffuse, and; hence, establishing a normal myocardium to determine a threshold is challenging. T1 mapping is more sensitive in detection of diffuse fibrosis, with higher native $\mathrm{T} 1$ values and elevated ECV described in $\mathrm{HCM}$. There is no correlation between LGE and T1 values, indicating that diffuse and regional fibrosis in HCM may be different entities [63].

\section{Athlete's heart}

Athlete's heart is a physiological hypertrophy seen as an adaptive response to the altered loading conditions of prolonged and intensive training. This has been reported in an estimated $2 \%$ of athletes [64]. At a cellular level, the normal structural organization and function are preserved, and the signalling pathway for this response is also distinct (IGF-1/IGF-1R/Akt axis) from pathological hypertrophy [65]. The LV geometry is variable- concentric hypertrophy in strength-trained athletes due to pressure overload; eccentric hypertrophy in endurance-trained athletes due to volume overload; and a mixed pattern of concentric-eccentric hypertrophy in others.

MRI shows hypertrophy, which may be concentric, eccentric or mixed concentric-eccentric (Fig. 14). Distinguishing

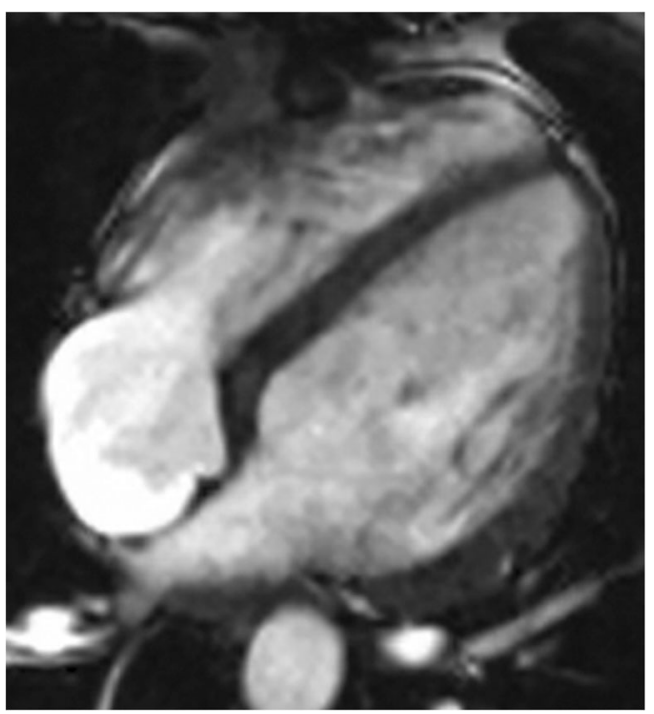

Fig. 14 4-chamber cine SSFP image shows mild LV thickening (15 mm) and mild LV dilation (EDV-200 ml; EDVi- $120 \mathrm{ml} / \mathrm{m} 2$ ) with normal systolic function (EF- 59\%). There was no abnormal LGE. This was diagnosed as athlete's heart. After cessation of athletic training for three months, the ventricular measurements returned to normal 


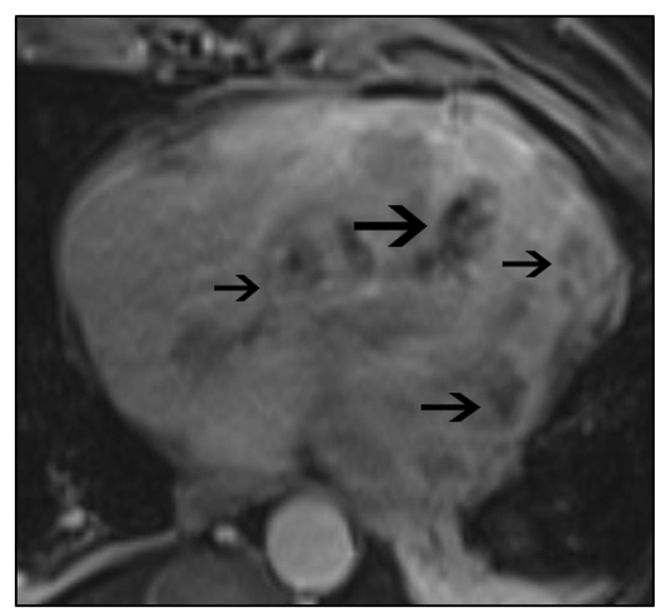

Fig. 15 4-chamber post-contrast T1-w gradient echo first-pass perfusion image shows heterogeneous myocardial enhancement with areas of necrosis in a patient with metastatic deposits from a primary angiosarcoma of the extremity (arrows)

athlete's heart from HCM is critical, since missing a diagnosis of HCM puts the athlete at risk of sudden death and an erroneous diagnosis of HCM will disqualify an otherwise eligible athlete from contact sports [66]. Features that distinguish athlete's heart from HCM are - the LVWT is $<15 \mathrm{~mm}$ in males and $13 \mathrm{~mm}$ in females; symmetrical thickening; normal septal/ lateral wall ratio; dilated cavity (but end-diastolic diameter $<$ $6.5 \mathrm{~cm}$ ), resulting in low RWM of $<1.16$; diastolic wall-tovolume ratio $<0.15 \mathrm{~mm} \times \mathrm{m} 2 \mathrm{x} \mathrm{ml}$ [66]; normal systolic function; normal/high diastolic function; and no LVOT gradient, SAM or perfusion defect. LGE is typically not seen unlike HCM; however, some studies have described LGE in12$50 \%$ of athletes $[67,68]$, with heterogeneous appearance, more commonly in the septum at the RV insertion site. Although the exact mechanism is unknown, theories include repetitive myocardial microtrauma, pulmonary artery pressure overload with dilated RV, genetic predisposition and silent myocarditis [68]. There may be proportional enlargement of the RV and atria. Clinical features that help in diagnosis are: absence of genetic abnormalities, milder ECG abnormalities (conduction delays, sinus bradycardia) and a peak oxygen uptake that is higher than expected for the age, gender and size. If the diagnosis of athlete's heart still remains indeterminate after using all the abovementioned criteria, it may be confirmed by regression of the structural changes following a period of deconditioning.

\section{Neoplastic infiltration}

Diffuse LV thickening may also be seen due to diffuse or multifocal neoplastic infiltration. This includes neoplasms such as metastases, lymphoma, leukemia or sarcoma. B cell lymphoma is the most common type of lymphoma to produce diffuse myocardial infiltration [69]. Although cardiac leukemic infiltration is present in $37-44 \%$ of patients with leukemia, clinically it is seen only in $1 \%$ of these patients [70]. While leukemic deposits may not be separately discerned, lymphomatous and metastatic deposits are often grossly seen. On black-blood MRI, LV thickening is present, which is more irregular and has heterogeneous signal intensities compared to the other causes of LV thickening. The imaging appearance mimics HCM in non-contrast sequences, but in contrastenhanced sequences, heterogeneous enhancement is noted (Fig. 15). Associated findings such as pericardial effusion may also be present.

\section{False positive hypertrophy}

Occasionally, pseudohypertrophy may be seen due to partial averaging of normal structures. This may be seen due to a prominent moderator band, which may give a false impression of septal hypertrophy (Movies 1 and 2).

\section{Summary}

MRI plays an important role in the comprehensive evaluation of patients with LV thickening. The unique tissue characterization capabilities of MRI enable establishing the aetiology of LV thickening, which is important for selecting the appropriate treatment. The high accuracy and reproducibility of MRI measured indices such as mass, volumes and function make it the ideal modality for serial follow-up in clinical scenarios and trials to evaluate treatment response. In addition, MRI also provides prognostic information.

\section{Compliance with ethical standards}

Conflict of interest The authors have no financial disclosure or conflict of interest.

Open Access This article is distributed under the terms of the Creative Commons Attribution 4.0 International License (http:// creativecommons.org/licenses/by/4.0/), which permits unrestricted use, distribution, and reproduction in any medium, provided you give appropriate credit to the original author(s) and the source, provide a link to the Creative Commons license, and indicate if changes were made.

\section{References}

1. Maceira AM, Mohiaddin RH (2012) Cardiovascular magnetic resonance in systemic hypertension. J Cardiovasc Magn Reson 14:28

2. Puntmann V, Jahnke C, Gebker R (2010) Usefulness of magnetic resonance imaging to distinguish hypertensive and hypertrophic cardiomyopathy. Am J Cardiol 106(7):1016-1022

3. Díez J (2007) Mechanisms of cardiac fibrosis in hypertension. J Clin Hypertens 9(7):546-550 
4. Levy D, Garrison RJ, Savage DD, Kannel WB, Castelli WP (1990) Prognostic implications of echocardiographically determined left ventricular mass in the Framingham Heart Study. N Engl J Med 322(22):1561-1566

5. Casale PN, Devereux RB, Milner M et al (1986) Value of echocardiographic measurement of left ventricular mass in predicting cardiovascular morbid events in hypertensive men. Ann Intern Med 105(2):173-178

6. Koren MJ, Devereux RB, Casale PN, Savage DD, Laragh JH (1991) Relation of left ventricular mass and geometry to morbidity and mortality in uncomplicated essential hypertension. Ann Intern Med 114(5):345-352

7. Olsen MH, Wachtell K, Hermann KL et al (2002) Is cardiovascular remodeling in patients with essential hypertension related to more than high blood pressure? A LIFE substudy. Am Heart J 144(3): 530-537

8. Devereux RB, Alonso DR, Lutas EM et al (1986) Echocardiographic assessment of left ventricular hypertrophy: Comparison to necropsy findings. Am J Cardiol 57(6):450-458

9. Armstrong AC, Gidding S, Gjesdal O et al (2012) LV mass assessed by echocardiography and CMR, cardiovascular outcomes, and medical practice. J Am Coll Cardiol Img 5(8):837-848

10. Senda Y, Tohkai H, Kimura M et al (1990) ECG-gated cardiac scan and echocardiographic assessments of left ventricular hypertrophy: reversal by 6 -month treatment with diltiazem. J Cardiovasc Pharmacol 16(2):298-304

11. Schlosser T, Mohrs OK, Magedanz A, Voigtländer T, Schmermund A, Barkhausen J (2007) Assessment of left ventricular function and mass in patients undergoing computed tomography (CT) coronary angiography using 64-detector-row CT: comparison to magnetic resonance imaging. Acta Radiol 48(1):30-35

12. Belge B, Coche E, Pasquet A, Vanoverschelde J-LJ, Gerber BL (2006) Accurate estimation of global and regional cardiac function by retrospectively gated multidetector row computed tomography: comparison with cine magnetic resonance imaging. Eur Radiol 16(7):1424-1433

13. La Gerche A, Taylor AJ, Prior DL (2009) Athlete's heart: the potential for multimodality imaging to address the critical remaining questions. JACC Cardiovasc Imaging 2:350-363

14. Grothue F et al (2002) Comparison of interstudy reproducibility of cardiovascular magnetic resonance with two-dimensional echocardiography in normal subjects and in patients with heart failure or left ventricular hypertrophy. Am J Cardiol 90(1):29-34

15. Tee M, Noble JA, Bluemke D (2013) Imaging techniques for cardiac strain and deformation: comparison of echocardiography, cardiac magnetic resonance and cardiac computed tomography. Expert Rev Cardiovasc Ther 11(2):221-231

16. Ginat DT, Fong MW, Tuttle DJ et al (2011) Cardiac imaging: Part 1, MR pulse sequences, imaging planes, and basic anatomy. Am J Roentgenol 197(4):808-815

17. Coelho-Filho OR, Rickers C, Kwong RY, Jerosch-Herold M (2013) MR myocardial perfusion imaging. Radiology 266(3):701-715

18. Karamitsos TD, Piechnik SK, Banypersard SM et al (2013) Noncontrast T1 mapping for the diagnosis of cardiac amyloidosis. J Am Coll Cardiol Img 6(4):488-497

19. Sado DM, White SK, Piechnik SK et al (2013) Identification and assessment of Anderson-Fabry disease by cardiovascular magnetic resonance noncontrast myocardial T1 mapping. Circ Cardiovasc Imaging 6(3):392-398

20. Sado DM, Maestrini V, Piechnik SK et al (2015) Noncontrast myocardial T1 mapping using cardiovascular magnetic resonance for iron overload. J Magn Reson Imaging 41(6):1505-1511

21. Maceira AM, Prasad SK, Khan M, Pennell DJ (2006) Normalized left ventricular systolic and diastolic function by steady state free precession cardiovascular magnetic resonance. J Cardiovasc Magn Reson 8(3):417-426
22. Dweck MR, Joshi S, Murigu T et al (2012) Left ventricular remodeling and hypertrophy in patients with aortic stenosis: insights from cardiovascular magnetic resonance. J Cardiovasc Magn Reson 14(1):50

23. Chirinos JA, Segers P, De Buyzere ML et al (2010) Left ventricular mass: allometric scaling, normative values, effect of obesity, and prognostic performance. Hypertension 56(1):91-98

24. Muiesan ML, Salvetti M, Monteduro C et al (2004) Left ventricular concentric geometry during treatment adversely affects cardiovascular prognosis in hypertensive patients. Hypertension 43(4):731738

25. Rodriguez JCL, Amadu AM, Dastidar AG, et al. (2016) Comprehensive characterization of hypertensive heart disease left ventricular phenotypes. Heart 0:1-9

26. Andersen K, Hennersdorf M, Cohnen M et al (2009) Myocardial delayed contrast enhancement in patients with arterial hypertension: initial results of cardiac MRI. Eur J RAdiol 71:75-81

27. Baumgartner H, Hung J, Bermejo J et al (2009) Echocardiographic assessment of valve stenosis: EAE/ASE recommendations for clinical practice. J Am Soc Echocardiogr 22(1):1-23

28. Smucker ML, Tedesco CL, Manning SB, Owen RM, Feldman MD (1988) Demonstration of an imbalance between coronary perfusion and excessive load as a mechanism of ischemia during stress in patients with aortic stenosis. Circulation 78(3):573-582

29. Azevedo CF, Nigri M, Higuchi ML et al (2010) Prognostic significance of myocardial fibrosis quantification by histopathology and magnetic resonance imaging in patients with severe aortic valve disease. J Am Coll Cardiol 56(4):278-287

30. Herrman S, Stork S, Neimann M et al (2011) Low-gradient aortic valve stenosis: myocardial fibrosis and its influence on function and outcome. J Am Coll Cardiol 58(4):402-412

31. Dweck MR, Joshi S, Murigu T et al (2011) Midwall fibrosis is an independent predictor of mortality in patients with aortic stenosis. J Am Coll Cardiol 58(12):1271-1279

32. Chaturvedi A, Hobbs SK, Ling FS et al (2016) MRI evaluation prior to Transcatheter Aortic Valve Implantation (TAVI): When to acquire and how to interpret. Insights Imaging 7(2):245-254

33. Katta R, Rathore V, Lip G (1997) Coarctation of aorta. J Hum Hypertens 11:537-538

34. Hom JJ, Ordovas K, Reddy GP (2008) Velocity-encoded cine MR imaging in aortic coarctation: functional assessment of hemodynamic events. Radiographics 28(2):407-416

35. Teis A, Sheppard MN, Alpendurada F (2010) Subaortic membrane: correlation of imaging with pathology. Eur Heart J 31(22):2822

36. O'Donnell DH, Abbara S, Chaithiraphan Vet al (2012) Cardiac MR imaging of non ischemic cardiomypathies: imaging protocols and spectra of appearances. Radiology 262(2):403-420

37. Seward JB, Casaclang-Verzosa G (2010) Infiltrative cardiovascular disease. Cardiomyopathies that look alike. J Am Coll Cardiol 55(17):1769-1779

38. Maceira AM, Prasad SK, Hawkins PN, Roughton M, Pennell DJ (2008) Cardiovascular magnetic resonance and prognosis in cardiac amyloidosis. J Cardiovasc Magn Reson 10:54

39. Syed IS, Glockner JF, Feng D et al (2010) Role of cardiac magnetic resonance imaging in the detection of cardiac amyloidosis. JACC Cardiovasc Imaging 3(2):155-164

40. Karamitsos TD, Piechnik SK, Banypersad SM et al (2013) Noncontrast T1 mapping for the diagnosis of cardiac amyloidosis. JACC Cardiovasc Imaging 6(4):488-497

41. Banypersad SM, Sado DM, Flett AS et al (2013) Quantification of myocardial extracellular volume fraction in systemic AL amyloidosis: an equilibrium contrast cardiovascular magnetic resonance study. Circ Cardiovasc Imaging 6(1):34-39

42. Seydelmann N, Wanner C, Stork S et al (2015) Fabry disease and the heart. Best Pract Res Clin Endocrinol Metabol 29:195-204 
43. Nakao S, Takenaka T, Maeda M et al (1995) An atypical variant of Fabry's disease in men with left ventricular hypertrophy. N Engl J Med 333(5):288-293

44. Sachdev B, Takenaka T, Teraguchi H et al (2002) Prevalence of Anderson-Fabry disease in male patients with late onset hypertrophic cardiomyopathy. Circulation 105(12):1407-1411

45. De Cobelli F, Esposito A, Belloni E, et al. (2009) Delayedenhanced cardiac MRI for differentiation of fabry's disease from symmetric hypertrophic cardiomyopathy. Am J Roentgenol 192(3)

46. Yang Z, McMahon CJ, Smith LR et al (2005) Danon disease as an underrecognized cause of hypertrophic cardiomyopathy in children. Circulation 112(11):1612-1617

47. Tada H, Harimura Y, Yamasaki H (2010) Utility of Real-time 3dimensional echocardiography and magnetic resonance imaging for evaluation of Danon disease. Circulation 121:e390-e392

48. Piotrowska-Kownacka D, Kownacki L, Kuch M et al (2009) Cardiovascular magnetic resonance findings in a case of Danon disease. J Cardiovasc Magn Reson 11:12

49. Dara B, Rusconi P, Fishman J (2011) Characteristic late gadolinium enhancement pattern on cardiac magnetic resonance imaging. Cardiol Young 21:707-709

50. Stranzinger E, Ensing G, Hernandez R (2008) MR findings of endocardial fibroelastosis in children. Pediatr Radiol 38:292-296

51. Rajiah P, Raza S, Saboo SS et al (2016) Update on the role of cardiac magnetic resonance in acquired on ischemic cardiomyopathies. J Thorac Imaging 31(6):348-366

52. Murphy CJ, Oudit GY (2010) Iron-overload cardiomyopathy: pathophysiology, diagnosis, and treatment. J Card Fail 16(11):888-900

53. Kremastinos DT, Farmakis D (2011) Iron overload cardiomyopathy in clinical practice. Circulation 124:2253-2263

54. Anderson LJ, Holden S, Davis B et al (2001) Cardiovascular T2 star magnetic resonance for the early diagnosis of myocardial iron overload. Eur Heart J 22(23):2171-2179

55. Vignaux O (2005) Cardiac sarcoidosis: spectrum of MRI features. Am J Roentgenol 194:249-254

56. Turkbey EB, McClelland RL, Kronmal RA et al (2010) The impact of obesity on the left ventricle. The Multi-Ethnic Study of Atherosclerosis (MESA). JACC Cardiovasc Imaging 3(3):266-274

57. Eguchi K, Boden-Albala B, Jin Z et al (2008) Association between diabetes mellitus and left ventricular hypertrophy in a multi-ethnic population. Am J Cardiol 101(12):1787-1791

58. Harrigan CJ, Appelbaum E, Maron BJ et al (2008) Significance of papillary muscle abnormalities identified by cardiovascular magnetic resonance in hypertrophic cardiomyopathy. Am J Cardiol 101(5):668-673
59. Maron M, Olivotto I, Harrigan C et al (2011) Mitral valve abnormalities identified by cardiovascular magnetic resonance represent a primary phenotypic expression of hypertrophic cardiomyopathy. Circulation 124(1):40-47

60. Rubinshtein R, Glockner JF, Ommen SR et al (2010) Characteristics and clinical significance of late gadolinium enhancement by contrast-enhanced magnetic resonance imaging in patients with hypertrophic cardiomyopathy. Circ Heart Fail 3(1): $51-58$

61. O'Hanlon R, Grasso A, Roughton M et al (2010) Prognostic significance of myocardial fibrosis in hypertrophic cardiomyopathy. J Am Coll Cardiol 56(11):867-874

62. Bruder O, Wagner A, Jensen CJ et al (2010) Myocardial scar visualized by cardiovascular magnetic resonance imaging predicts major adverse events in patients with hypertrophic cardiomyopathy. $\mathrm{J}$ Am Coll Cardiol 56(11):875-887

63. Ellims AH, Iles LM, Ling LH et al (2012) Diffuse myocardial fibrosis in hypertrophic cardiomyopathy can be identified by cardiovascular magnetic resonance, and is associated with left ventricular diastolic dysfunction. J Cardiovasc Magn Reson 14:76

64. Pelliccia A, Maron BJ, Spataro A, Proschan MA, Spirito P (1991) The upper limit of physiologic cardiac hypertrophy in highly trained elite athletes. N Engl J Med 324(5):295-301

65. Fagard R (2012) Athlete's heart or hypertrophic cardiomyopathy. Heart Metab 56:14-19

66. Petersen SE, Selvanayagam JB, Francis JM et al (2005) Differentiation of athlete's heart from pathological forms of cardiac hypertrophy by means of geometric indices derived from cardiovascular magnetic resonance. J Cardiovasc Magn Reson 7(3):551558

67. Breuckmann F, Möhlenkamp S, Nassenstein K et al (2009) Myocardial late gadolinium enhancement: prevalence, pattern, and prognostic relevance in marathon runners. Radiology 251(1): $50-57$

68. Van de Schoor FR, Aengevaeren VL, Hopman MTE (2016) Myocardial fibrosis in athletes. Mayo Clin Proc 91(11):1617-1631

69. Chalabreysse L, Berger F, Loire R, Devouassoux G, Cordier J-F, Thivolet-Bejui F (2002) Primary cardiac lymphoma in immunocompetent patients: a report of three cases and review of the literature. Virchows Arch 441:456-461

70. Mateen FJ, Harding SR, Saxena A (2006) Extensive myocardial infiltration by hemopoietic precursors in a patient with myelodysplastic syndrome. BMC Blood Disord 6:4 